\title{
High-latitude HF Doppler observations of ULF waves: 2. Waves with small spatial scale sizes
}

\author{
D. M. Wright, T. K. Yeoman \\ Department of Physics and Astronomy, University of Leicester, University Road, Leicester, LE1 7RH, UK
}

Received: 25 August 1998 / Revised: 7 January 1999 / Accepted: 29 January 1999

\begin{abstract}
The DOPE (Doppler Pulsation Experiment) HF Doppler sounder located near Tromsø, Norway (geographic: $69.6^{\circ} \mathrm{N} 19.2^{\circ} \mathrm{E} ; L=6.3$ ) is deployed to observe signatures, in the high-latitude ionosphere, of magnetospheric ULF waves. A type of wave has been identified which exhibits no simultaneous ground magnetic signature. They can be subdivided into two classes which occur in the dawn and dusk local time sectors respectively. They generally have frequencies greater than the resonance fundamentals of local field lines. It is suggested that these may be the signatures of high- $m$ ULF waves where the ground magnetic signature has been strongly attenuated as a result of the scale size of the waves. The dawn population demonstrate similarities to a type of magnetospheric wave known as giant (Pg) pulsations which tend to be resonant at higher harmonics on magnetic field lines. In contrast, the waves occurring in the dusk sector are believed to be related to the storm-time Pc5s previously reported in VHF radar data. Dst measurements support these observations by indicating that the dawn and dusk classes of waves occur respectively during geomagnetically quiet and more active intervals.
\end{abstract}

Key words. Ionosphere (auroral ionosphere; ionosphere-magnetosphere interactions) ·

Magnetospheric physics (MHD waves and instabilities)

\section{Introduction}

An important coupling mechanism between the magnetosphere and the ionosphere is ultra low frequency (ULF) waves, since they transfer both energy and

Correspondence to: D. M. Wright momentum. These processes are most significant in the high-latitude ionosphere, where the magnetosphereionosphere interaction is strongest. The waves also act as an important diagnostic of magnetospheric morphology and dynamics. The ionosphere determines the boundary conditions (e.g. Yeoman et al., 1990) for magnetospheric magnetohydrodynamic (MHD) wave modes and hence controls the transfer of energy and momentum. It also modifies the magnetospheric wave signature, leading to rotation and attenuation of the wave magnetic signature detected on the ground (e.g. Hughes and Southwood, 1976; Hughes, 1983). The ionospheric signature of ULF waves is thus an important topic of study.

Field line resonances (FLRs; e.g. Southwood, 1974; Chen and Hasagawa, 1974) are fairly well understood and their ionospheric signatures have been observed by VHF (e.g. Walker et al., 1979; Yeoman et al., 1990) and HF (e.g. Ruohoniemi et al., 1991; Yeoman et al., 1997) radars in the E- and F-regions respectively. They exhibit small effective azimuthal wave numbers $(m)$ typically in the range 0-20, which is equivalent to azimuthal wavelengths $\left(\lambda_{\mathrm{az}}\right)$ of greater than $685 \mathrm{~km}$ at the latitude of Tromsø $\left(69.6^{\circ} \mathrm{N}\right)$. Non-resonant ULF wave features have also been observed by VHF radars (e.g. Allan et al., 1982, 1983).

Fenwick and Villard (1960) first suggested that geomagnetic variations might be associated with observed shifts in the frequency of ionospherically reflected radio signals collected from an HF Doppler sounder. Since then, short-period oscillations in the frequency of HF signals have been correlated with magnetospheric ULF pulsations measured by ground magnetometers (e.g. Jacobs and Watanabe, 1966; Klostermeyer and Röttger, 1976; Tedd et al., 1989; Menk, 1992). HF Doppler sounders offer an important experimental technique for investigating the ionospheric signatures of magnetospheric ULF waves. This type of radio diagnostic provides measurements of the ionosphere with high spatial and temporal resolution (Wright et al., 1997). The Doppler Pulsation Experiment (DOPE), 
constructed in Leicester, UK, is deployed near Tromsø, Norway (geographic: $69.6^{\circ} \mathrm{N} 19.2^{\circ} \mathrm{E}$; geomagnetic: $67.0^{\circ} \mathrm{N} 117.0^{\circ} \mathrm{E} ; L=6.3$ ) close to the European Incoherent Scatter (EISCAT) radar facility (e.g. Rishbeth and Williams, 1985). Comparison of DOPE measurements with EISCAT data from suitable common and special programs facilitates the investigation of the ionospheric boundary conditions for MHD wave modes in the magnetosphere. In addition, height profiles of ULF wave signatures are possible and the observations can be related to existing theoretical models and measurements made with other ground-based instruments (Wright et al., 1998). In recent years, an attempt has been made to model the mechanisms involved in generating the ionospheric signatures observed by $\mathrm{HF}$ Doppler sounders (Poole et al., 1988; Sutcliffe and Poole, 1989, 1990; Sutcliffe, 1994).

This study accompanies that by Wright et al. (1997; henceforth to be known as paper 1) which described DOPE observations of resonant ULF waves. The current study details ionospheric signatures of ULF waves which have little or no associated ground magnetic signature and are considered to be the result of waves with small spatial scale sizes. These waves are visible to DOPE due to its high spatial resolution in the ionosphere when compared to that of a ground magnetometer which is limited by spatial integration effects (e.g. Hughes, 1983). A clear example of this spatial integration has recently been described by Yeoman et al. (1997) where the CUTLASS radar provided measurements of a ULF wave with a spatial and temporal resolution better than previously achieved. The data demonstrated the evolution of a magnetospheric cavity resonance as it first became a transient feature with a latitude-dependent wave period and subsequently evolved into a very narrow field line resonance. This latter manifestation of the wave had a very small-scale size of $60 \mathrm{~km}$ and was very strongly attenuated in the ground magnetometer data.

High- $m$ pulsations are a topic of considerable interest at present, in both theoretical and experimental studies. It is now widely accepted that a source of these ULF waves exists in drifting energetic particle fluxes. Particles of this type entering the Earth's near geospace from the geotail will undergo gradient curvature drift and, thus, move around the Earth constituting part of the global ring current. The drifting particles can drive MHD wave modes through wave-particle interactions, leading to perturbations in the electric and magnetic fields in the ionosphere (e.g. Hughes, 1983). Recently there have been a number of studies attempting to explain the occurrence and characteristics of high- $m$ field line resonances in HF radar observations (Fenrich et al., 1995; Fenrich and Samson, 1997). These waves occur simultaneously with low- $m$ field line resonances, at the same wave frequency and on similar $L$-shells but westwards of the low- $m$ resonance location. A nonlinear Kelvin-Helmholtz instability has been proposed (Allan and Wright, 1997; Mann, 1998) as a coupling mechanism between the low- $m$ wave guide modes (e.g. Walker et al., 1992; Wright, 1994) and the high- $m$ resonances.
The velocity shear of the low- $m$ resonant wave is thought to drive the instability which sets up a spectrum of high- $m$ waves, one of which begins to grow in amplitude. Unstable distributions of westward drifting ions can then provide the energy to amplify the high- $m$ seed waves which are expected (Allan and Wright, 1997) and observed (e.g. Fenrich et al., 1995) to occur in the dawn and dusk sectors.

Two categories of particle-driven waves have been particularly intensively studied with ground-based data: the $\mathbf{E} \times \mathbf{B}$ drift imposed on the ionospheric plasma by the ULF wave is observed by VHF coherent radars (e.g. Yeoman et al., 1992), such as SABRE (Sweden and Britain Radar Experiment; Nielsen et al., 1983). This is believed to be the dominant mechanism which leads to detection of ULF wave signatures in the backscatter received by these systems in the dusk local time sector. These "storm time Pc5s" seen in STARE (Scandinavian Twin Auroral Radar Experiment; Greenwald et al., 1978) data (Allan et al., 1982, 1983) are compressional waves of high- $m$ number and period in the Pc5 range which are associated with the suggested source mechanism.

The second class of particle driven ULF waves of interest are giant 'Pg' pulsations which have been observed on the ground as well as by orbiting satellites at times when geomagnetic conditions are quiet. A paper by Hughes et al. (1979) describes multiple satellite observations of a short period (55 s) compressional wave at geostationary orbit. These authors deduced that the wave was a second harmonic standing wave which led to their interpretation of the driving mechanism being a bounce resonance interaction with ring current protons. The wave number, $m$, was calculated to be 100 . The harmonic mode of this wave is in agreement with the observations made by several other authors (e.g. Annexstad and Wilson, 1968). However, there are others (e.g. Green, 1979) who consider that the waves are odd mode. More recently, Chisham and Orr (1991) presented a statistical study of 34 of these events observed on the EISCAT magnetometer cross in northern Scandinavia. They found a peak in occurrence of these waves in the dawn/prenoon sector and no events were observed in the afternoon. They deduced that Pgs are second harmonic (even mode) standing wave oscillations. The average value of the azimuthal wave number $(m)$ was $\sim 26$ for the 34 events.

\section{Instrumentation}

\subsection{The DOPE sounder}

The DOPE (Doppler Pulsation Experiment) sounder consists of a frequency stable transmitter (Chapman, 1995, 1997a; Wright et al., 1997) and a receiver (Chapman, 1997b) which have a ground-separation of about $50 \mathrm{~km}$, giving a near vertical radio path roughly along a magnetic meridian. A fixed frequency $(4.45 \mathrm{MHz})$ continuous wave signal is radiated and, after reflection in the F-region ionosphere, received at the ground. The 
Doppler technique utilises the fact that variations in the refractive index or bulk motion of the plasma along the path of the radio wave cause small shifts in the received frequency, due to changes in the phase path of the wave. Thus, the frequency shift, $\Delta f$, is given by

$$
\Delta f=-\frac{1}{\lambda} \frac{\mathrm{d} P}{\mathrm{~d} t}
$$

where $P$ is the phase path of the signal and $\lambda$ is the wavelength. In the case where the phase path is not affected by changes in refractive index, this can be interpreted as an equivalent vertical bulk motion of the reflection point (plasma) with a velocity, $v$, using, for a vertical incidence sounder, the relation

$$
\Delta f=-2 \frac{v}{c} f
$$

where $c$ is the speed of light and $f$ is the sounding frequency.

The spatial resolution of the DOPE has been shown (Wright et al., 1997) to be of the order of $4 \mathrm{~km}$ (for an F-region reflection height of $250 \mathrm{~km}$ and a sounder frequency of $4.45 \mathrm{MHz}$ ). In addition, the sensitivity of the instrument increases as the sounding frequency approaches the local ionospheric critical frequency. More detailed information regarding the DOPE system and the Doppler technique are available in Wright (1996) and in paper 1.

\subsection{Data acquisition and analysis}

The DOPE twin channel receiver enables O- and Xmode signals to be resolved (Chapman, 1997b). The receiver mixes the signal input frequency down to a baseband level which represents the offset from the diagnostic frequency of $4.45 \mathrm{MHz}$. So far as the authors are aware, this system is the first to offer continuous $\mathrm{O}$ and X-mode observations of ULF wave signatures at high latitudes. There are few previous observations of ULF wave signatures with HF sounders utilising O-X mode discrimination and those that do exist occurred at mid and low latitudes (e.g. Jarvis and Gough, 1988; Sutcliffe and Poole, 1984; see also paper 1).

The raw data from DOPE are passed through a fast Fourier transform (FFT) routine in order to produce a "Doppler trace" such as those reproduced in the top two panels of Fig. 1. The temporal resolution of these data is $12.8 \mathrm{~s}$. Spectral components with values above some threshold (usually $50 \%$ of the peak value) are included on the Doppler trace and these represent the diagnostic signal frequency shift. Further details on the analysis techniques utilised here are reported in paper 1.

\subsection{Ground magnetometers}

In addition to the Doppler data presented in this work, data from two ground magnetometer stations in northern Scandinavia are included. These stations are Tromsø (TRO), Norway and Sodankylä (SOD; geographic: $67.4^{\circ} \mathrm{N}, 26.6^{\circ} \mathrm{E}$ ), Finland which are both part of the IMAGE magnetometer chain (e.g. Lühr, 1994). These data have a time resolution of $10 \mathrm{~s}$. The resolution of magnetic field perturbation magnitude measured by TRO is $1 \mathrm{nT}$ and that of SOD is considerably better at $0.01 \mathrm{nT}$. Spatially separated stations offer the possibility to determine the latitudinal phase change of a ULF wave as well as its azimuthal phase change, characterised by the effective azimuthal wave number, $m$, the number of degrees of change in phase per degree of longitude on the Earth's surface. Due to the way in which ground magnetometers integrate information over an area with a scale length of the order of the Eregion height ( $120 \mathrm{~km}$; Hughes and Southwood, 1976), phase mixing of signals from adjacent regions in the ionosphere result in the attenuation of waves observed on the ground with high $m$-values (corresponding to small azimuthal scale lengths).

\section{Results}

\subsection{DOPE observations of ULF waves}

A commonly occurring type of ULF wave signature observed by the DOPE sounder are those with no associated ground magnetic signature detected by nearby magnetometers. Figure 1 illustrates an event of this type. The top two panels display $\mathrm{O}$ - and $\mathrm{X}$-mode Doppler traces (Fig. 1a, b respectively) for an event which occurred on 2nd September, 1995. The signature commenced sometime before 0300 UT and was observed to continue until around 0450 UT. In the interval 04000430 UT seventeen wave cycles are clearly visible in the $\mathrm{X}$-mode Doppler trace and these are amplitude modulated into wave packets. The corresponding O-mode signature although apparent is slightly more noisy and an interfering signal is seen encroaching into the true signal (which has an offset of about $+3 \mathrm{~Hz}$ ) at around 0500 UT. The amplitude of the wave maximises at about 0400 UT with a Doppler shift of $4 \mathrm{~Hz}$ peak to peak, which is equivalent (Eq. 2) to a vertical oscillation of the reflection level with a velocity of $136 \mathrm{~ms}^{-1}$. The lower two panels of Fig. 1 display the $\mathrm{X}$ - and Y-component (Fig. 1c, d respectively) magnetometer data from Tromsø (TRO), Norway and Sodankylä (SOD), Finland. It is evident from these data that no simultaneous ground magnetic signature was associated with this event.

By selecting the Fourier frequency component of maximum power in the HF Doppler data in Fig. 1a, b a single time series was obtained for each of the signatures. The power spectra of those time series for the interval 0355-0422 UT are given in Fig. 2. It clearly shows a peak spectral component in both the $\mathrm{O}$ - and $\mathrm{X}$ mode data at $9.2 \mathrm{mHz}$ (equivalent to a period of $109 \mathrm{~s}$ ) which is in the Pc4 (Jacobs et al., 1964) frequency range. It should be noted that events are commonly observed when pulsation activity was apparent in the TRO magnetometer data. However, in this event there was no signature present at the same frequency as that observed by DOPE in the F-region ionosphere or at any related harmonic frequency. 


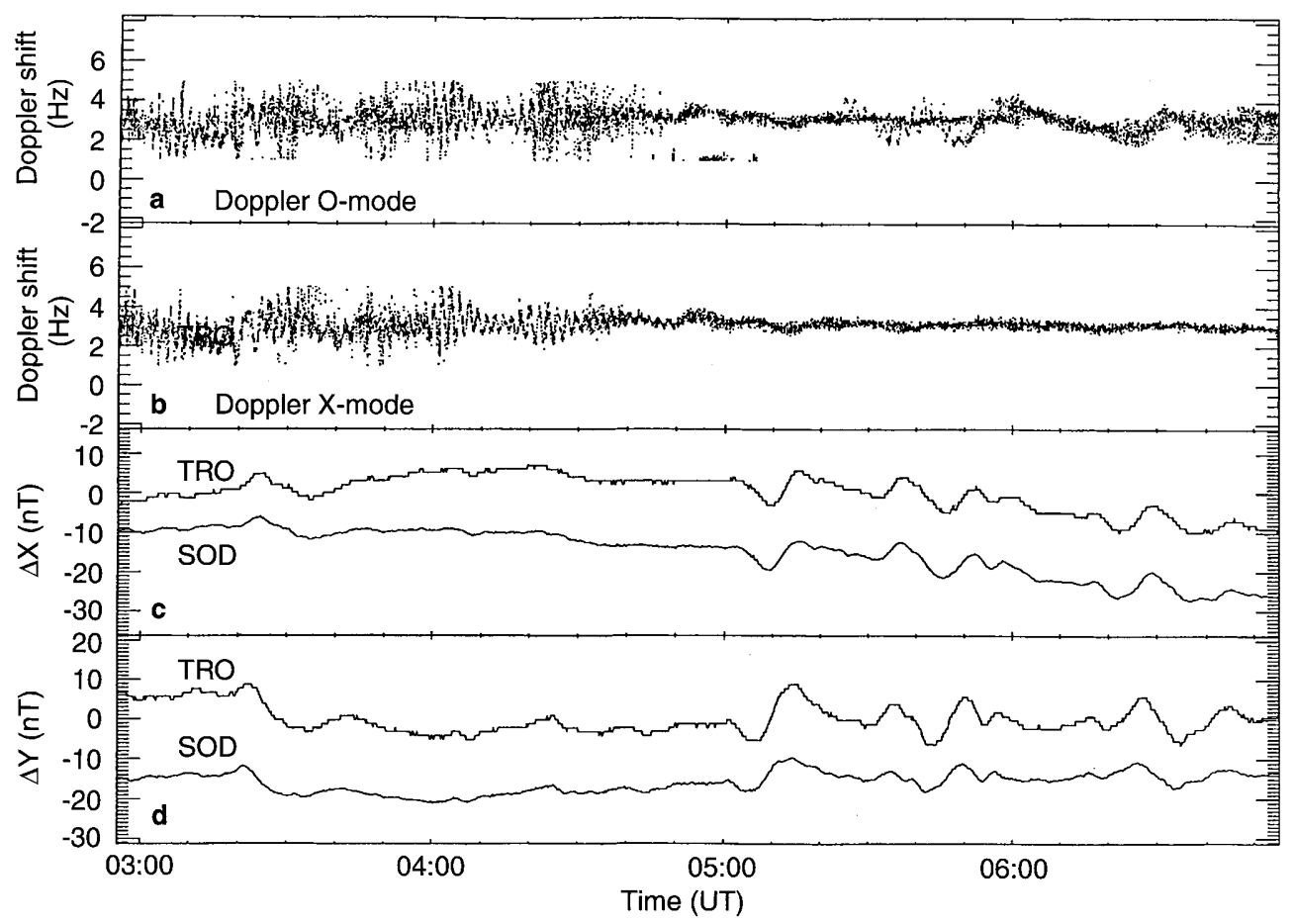

Fig. 1a-d. HF Doppler a O- and b X-mode traces for the ULF wave event which occurred on 2 September, 1995. A simultaneous ground magnetic signature is absent in both $\mathbf{c} \mathrm{X}$ - and $\mathbf{d} \mathrm{Y}$ component data recorded at Tromsø $(T R O)$, Norway and Sodankylä $(S O D)$, Finland

\subsection{Analysis of the events}

A section of the DOPE data set, the interval May 1995 to February 1996, has been examined in detail in order to determine the characteristics of the ULF signatures of the type shown in Fig. 1. This type of wave shall henceforth be known as an uncorrelated event since they are observed to have no correlated ground magnetic signature. Another type of signature, known as correlated events are also commonly identified in the data and these have previously been reported (Wright et al., 1997, 1998). 159 events including both types were recorded by DOPE in the aforementioned interval. The current study is only concerned with uncorrelated signatures. In order to be able to perform adequate frequency analyses on a Doppler trace, only the clearest (i.e. least noisy)

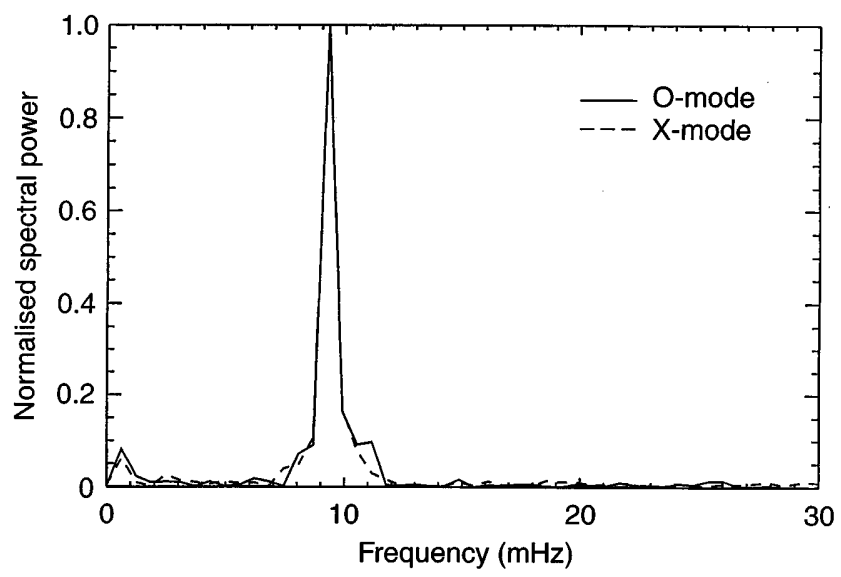

Fig. 2. Normalised power spectra of the Doppler O- and X-mode time series for the interval 0355-0422 UT on 2 September as displayed in Fig. 1 signatures have been selected. So far 12 of these have been analysed in detail.

Figure 3 displays some of the characteristics of the 12 events. The panels give the measured wave frequency (top), diurnal occurrence distribution (middle) and the relative phase difference $\left(\Delta \varphi_{\mathrm{O}-\mathrm{X}}\right)$ between the signatures observed in the $\mathrm{O}$ - and $\mathrm{X}$-mode signals (bottom). A negative value of $\Delta \varphi_{\mathrm{O}-\mathrm{X}}$ indicates that the $\mathrm{O}$-mode signature was leading in phase. The observed wave frequencies were in the range $4-18 \mathrm{mHz}$ (periods 55 $250 \mathrm{~s}$ ) with a frequency of $\sim 10 \mathrm{mHz}(100 \mathrm{~s})$ occurring most commonly. Thus many of these signatures have frequencies in the range of Pc4 pulsations, which exhibit periods between 40 and $150 \mathrm{~s}$ (Jacobs et al., 1964).

The diurnal occurrence distribution of the 12 selected uncorrelated events, which is given in Fig. 3b, is concentrated into two groups, one in the morning between 4-10 UT and another in the interval 14-17 UT. None of the events are identified around 12 UT. Most uncorrelated events exhibit little or no phase difference between the $\mathrm{O}$ - and $\mathrm{X}$-mode Doppler signatures (Fig. 3c). Observed values of $\Delta \varphi_{\mathrm{O}-\mathrm{X}}$ range from $-20^{\circ}$ to $45^{\circ}$ but most events exhibit a relative phase around $0^{\circ}$ suggesting that effects of the ULF waves were observed simultaneously at both the $\mathrm{O}$ - and $\mathrm{X}$-mode reflection points despite being spatially separated (in height and along a magnetic meridian) by up to $50 \mathrm{~km}$ (Wright et al., 1997, 1998).

\section{Discussion}

\subsection{Alternative causes of the uncorrelated waves}

In order to establish that the uncorrelated events observed by DOPE are signatures caused by incident 

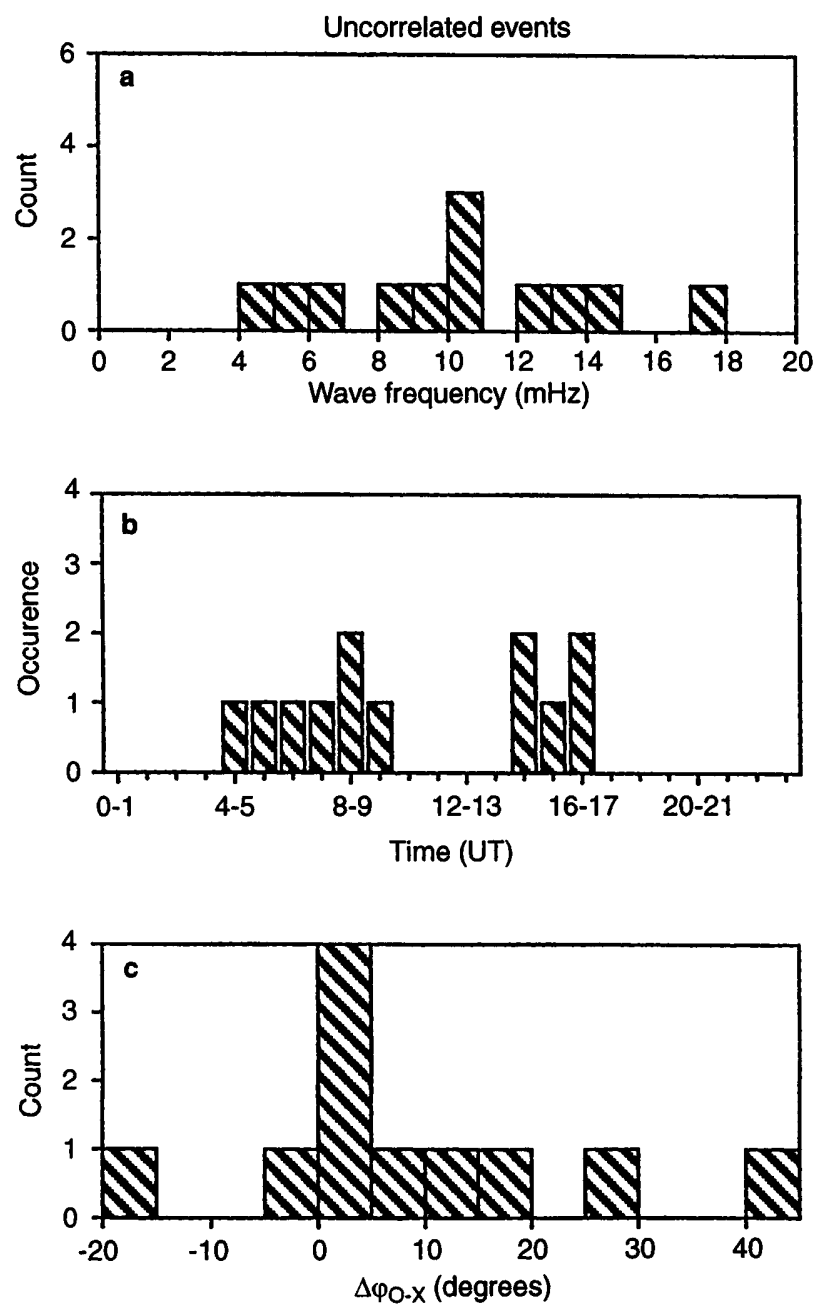

Fig. 3a-c. Characteristics of the uncorrelated wave signatures for the 12 events: a ULF wave frequency, $\mathbf{b}$ diurnal occurrence and $\mathbf{c} \Delta \varphi_{\mathrm{O}-\mathrm{X}}$, the relative phase of the signatures in the $\mathrm{O}$ - and $\mathrm{X}$-mode HF Doppler data

magnetospheric ULF waves, other possible sources of Doppler wave signatures with periods in the ULF range must be considered. Georges (1973) presented a detailed discussion of infrasonic waves which have periods in the range $2-5 \mathrm{~min}(3-8 \mathrm{mHz})$. These were briefly mentioned by Klostermeyer and Röttger (1976) as a possible cause of 2-4 min oscillations apparent in their F-region HF Doppler observations. Such waves are generated in regions of convective storms and propagate vertically upwards with phase velocities of $500-600 \mathrm{~ms}^{-1}$ (Georges, 1973) and lead to signatures in HF Doppler but not in ground magnetograms. Thus they are a possible source of uncorrelated waves. However, several characteristics of these waves and of the data presented here strongly suggest that they are not responsible for the uncorrelated events. Infrasonic waves appear to be restricted almost exclusively to occurrence in the evening whereas the uncorrelated events observed in this study occur in the dawn and postnoon sectors. Georges (1973) also stated that all significant observations of infrasonic waves have only occurred in the central United States of America. Unsuccessful attempts were made to detect them on the east coast of the USA in Florida.
Furthermore, the phase velocity and frequency of the waves (typically $4 \mathrm{mHz}$ ) suggest a vertical wavelength of the order of $120 \mathrm{~km}$. Thus, the relative phase between the signatures at the $\mathrm{O}$ - and $\mathrm{X}$-mode reflection heights, which may have a vertical separation of up to $50 \mathrm{~km}$ when the sounding frequency is close to the critical frequency, would essentially be randomised due to the range of phase speeds and frequencies. However, the uncorrelated events in this study seem to mainly have zero O-X mode phase difference. It seems unlikely, therefore, that infrasonic waves have any bearing on the present observations.

Menk (1992) listed some possible ways that Doppler signatures, with frequencies in the ULF range, might be generated by a method other than a pulsation. It was suggested that multi-mode HF wave beating might occur between either multi-hop rays or between a ground wave and an ionospherically reflected wave. However, the experimental arrangement of DOPE, where the signal phase path is near vertical and mountains between the transmitter and receiver block the ground wave path, makes it highly unlikely that either of these effects are responsible for the Doppler signatures observed in the DOPE experiment. In addition, the suggestion by Menk (1992) that O-X mode polarisation interference might result in a beat at ULF wave frequencies can be dismissed because at high latitudes such interference is negligible compared to that at low latitudes (e.g. Toman, 1967; Reddi and Rao, 1967) where Menk (1992) made measurements.

After careful consideration of the aforementioned effects, we conclude that the observed signatures are the result of ULF waves of magnetospheric origin and that this work provides the first confirmed observations, at high latitudes, of truly uncorrelated MHD waves with HF Doppler sounder signatures. Similar signatures have previously been observed at mid and low latitudes by other sounders (e.g. Klostermeyer and Röttger, 1976; Menk, 1992) but these were not confirmed to be the result of MHD waves of magnetospheric origin.

\subsection{Contrasts with resonant signatures}

Paper 1 describes signatures, also recorded by DOPE, associated with low- $m$ (large spatial scale) ULF waves which manifested themselves as field line resonances and exhibited simultaneous HF Doppler and ground magnetic signatures. The unique characteristics of the uncorrelated waves will be made most apparent by direct comparison with the results of paper 1 . Thus Fig. 4 is reproduced from this paper and provides a means of comparison with Fig. 3.

The measured wave frequencies (Fig. 3a) of the uncorrelated events are mainly in the Pc4 frequency range $(\geq 6.7 \mathrm{mHz}$; Jacobs et al., 1964) which is in contrast to those of the correlated waves which generally were identified as Pc5s. Poulter et al. (1984) presented (see their Fig. 2) the periods associated with field line resonances as a function of geomagnetic latitude derived from STARE VHF radar observations. The range of 

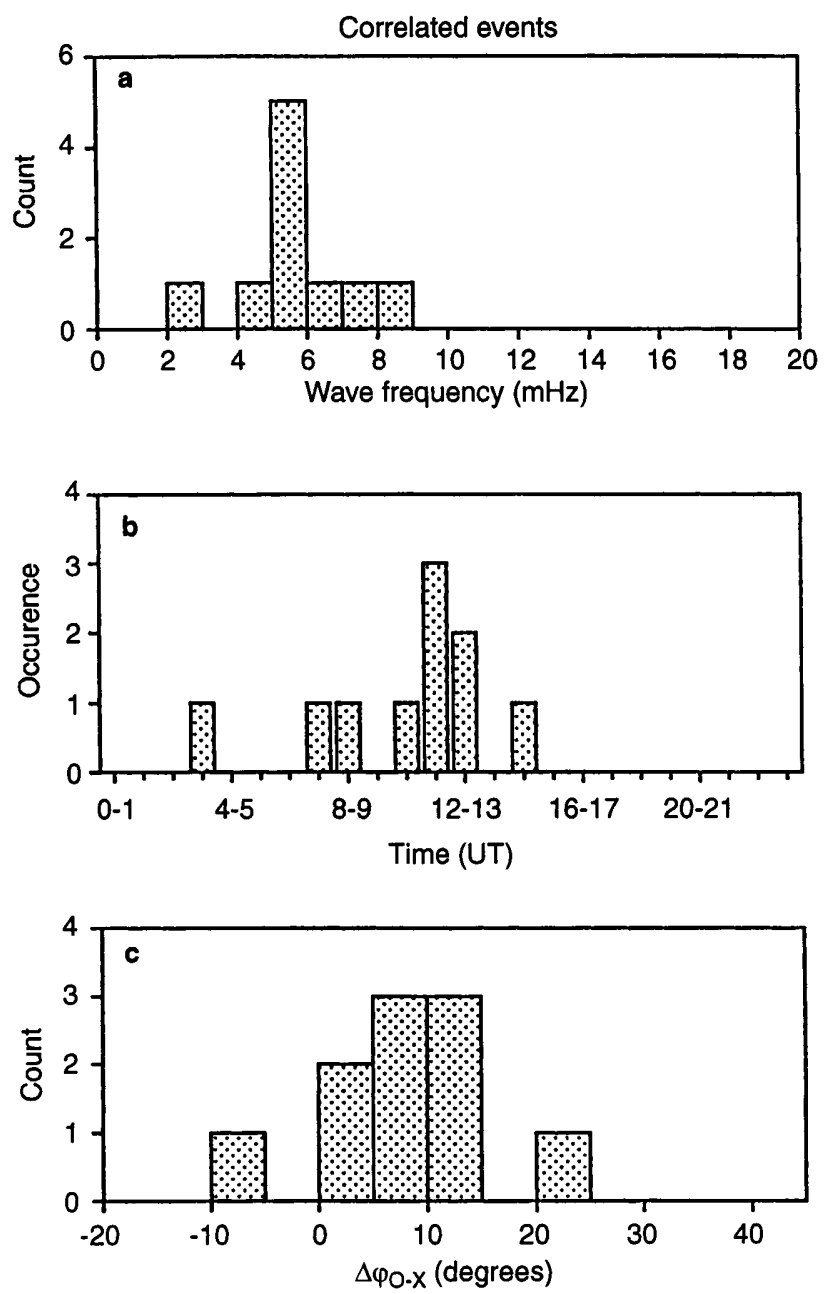

Fig. 4a-c. Characteristics of the correlated wave signatures for the 10 events studied in Paper 1 (see main text): a ULF wave frequency, b diurnal occurrence and $\mathbf{c} \Delta \varphi_{\mathrm{O}-\mathrm{X}}$, the relative phase of the signatures in the O- and X-mode HF Doppler data

periods associated with low $m$ field line resonances at the latitude of Tromsø were about 200-590 s (equivalent to frequencies $1.7-5 \mathrm{mHz}$ ), which is essentially the whole Pc5 range. This suggests that the waves reported here are not resonant features, unlike the waves described in paper 1 (Fig. 4a).

The diurnal occurrence distribution of the 12 selected uncorrelated events, which is given in Fig. 3b, is concentrated into two groups, one in the morning, between 4-10 UT, and another in the interval 14-17 UT. None of the events are identified around 12 UT. This compares with the distribution for resonant signatures (Fig. 4b) which occur dominantly in a region with a peak around 12 UT (just after local noon).

A characteristic feature of a field line resonance is that the phase of the wave changes by $180^{\circ}$ at either side, in a magnetic meridian, of the resonance region (Southwood, 1974), with the phase poleward of the resonant field line lagging that equatorward of it (e.g. Orr, 1984). Due to their differing propagation characteristics the $\mathrm{O}$ - and X-mode signals of the Doppler sounder at Tromsø deviate north and south, respective- ly. The relative phase, $\Delta \varphi_{\mathrm{O}-\mathrm{X}}$, of the ground correlated signatures in the $\mathrm{O}$ - and $\mathrm{X}$-mode signals received by DOPE (Fig. 4c; also see paper 1) are thus consistent with the characteristic latitudinal phase signatures of field line resonances. However, in most cases, the $\mathrm{O}$ - and $\mathrm{X}$-mode signatures of the uncorrelated waves are generally in phase (Fig. 3c). This suggests that a resonant phase structure is absent during these events.

\subsection{The morning population}

Giant (Pg) pulsations occur most commonly in the morning sector in the 02-07 LT interval, peaking in the range 03-06 LT, (Green, 1979; Chisham and Orr, 1991) with few observations in the afternoon. Bounce resonance wave particle interactions in the quiet time ring current have been suggested as the likely mechanism causing this type of ULF wave (e.g. Glassmeier, 1980). Kokubun (1985) reported that a subset of the azimuthally polarised Pc5 waves observed at geostationary orbit by the GOES2 satellite did not have an associated ground magnetic signature at the foot point of the satellite field line. The diurnal distribution of these events peaked around 3-5 LT. These spacecraft observations and the prenoon events presented here are both reminiscent of the distribution for giant pulsations. The high $m$-numbers associated with these waves (e.g. Chisham and Orr, 1991) would explain why a ground magnetic signature is not observed. Ionospheric screening effects shield high- $m$ pulsations from detection by ground magnetometers (Hughes and Southwood, 1976) which integrate data over an area with a spatial scale size of about $120 \mathrm{~km}$ (e.g. Hughes, 1983). Thus a Pgtype wave with a particularly high $m$-number or a relatively small magnetospheric amplitude is likely to remain undetected by a ground magnetometer.

\subsection{Observations in the post-noon sector}

The afternoon population of events presented here (Fig. 3b) are considered to be the results of a different type of signature. ULF waves with a high $m$-number have been observed in radar auroral backscatter power by SABRE without a concurrent signature on ground magnetometers (Yeoman et al., 1992). The occurrence distribution for these events, which had equatorward phase propagation, peaked at about 18 UT (about 20 MLT; Mao Tian et al., 1991). In addition, the diurnal distribution of storm time Pc5s, high- $m$ compressional waves which also tend to have no ground magnetic signature, observed on the STARE radar (Allan et al., 1983) occur in the 14-20 LT interval, having peak occurrence around 17-18 LT. Using data from the GOES2 and GOES3 satellites, Kremser et al. (1981) studied ULF wave signatures in energetic particle flux oscillations associated with storm time Pc5s. They observed that most events occurred between 14 and 20 LT. These types of waves have been associated with instabilities in drifting ring current protons and would 
be consistent with the afternoon population of uncorrelated waves shown in Fig. 3 b.

\subsection{Associated DST measurements}

Dst is a global index which is a measurement of the deviation of the low-latitude magnetic field from its mean value as a result of variations in the equatorial ring current. The magnetic field associated with the current generated by an injection of particles on the nightside, which subsequently drift around the Earth, opposes the Earth's own magnetic field. Hence, such an injection will have an associated depression in Dst. Figure 5 gives the results of a superposed epoch analysis of Dst magnitude around each of the events presented in the current study and in paper 1 . That is, the Dst values for $12 \mathrm{~h}$ before and $6 \mathrm{~h}$ after the start of each wave signature are averaged in one hour bins in order to determine the mean variation. The upper panel (Fig. 5a) illustrates the Dst values around the correlated events and the middle and lower panels (Fig. 5b, c) represent, respectively, the magnitude of the index associated with the uncorrelated events observed in the dawn and dusk sectors. Dst is slightly depressed during intervals when correlated events occur. However, there seems to be some difference in Dst between uncorrelated signatures which commence in the prenoon and postnoon sectors. Figure $5 \mathrm{~b}$ suggests that the prenoon events occurred when geomagnetic activity was low or at least there were no significant plasma injections associated with these times. This supports the idea that these events may be associated with particle driven quiet-time (Green, 1979) giant $(\mathrm{Pg})$ pulsations. The postnoon intervals, however, on average had more negative changes in $D s t$ around the times that the waves were observed. The changes in the index are larger here than for the times when correlated events occurred. This tends to support the likelihood that postnoon, but not prenoon, uncorrelated waves are associated with particle injections under geomagnetically active conditions and may be the same type of event as the storm time Pc5s observed with the STARE radar (Allan et al., 1982, 1983) and the equatorward propagating events identified in SABRE radar data by Yeoman et al. (1992) (see their Fig. 11). Both of these types of VHF radar signature had high measured $m$ values and were either attenuated in ground magnetometer data or not observed at all.

\subsection{Estimating the scale size of the ULF waves}

The spatial resolution of the SABRE and STARE VHF radars is $\sim 15 \mathrm{~km}$ and thus these instruments are much more sensitive to small-scale waves than ground magnetometers (Sect. 2.3; Hughes, 1983). The spatial resolution of the DOPE sounder has been estimated to be $\sim 4 \mathrm{~km}$ (Sect. 2.1; Wright et al., 1997) which would suggest that it has the ability to detect ULF wave signatures with even smaller spatial scale sizes. The field line resonance observed by the CUTLASS HF radar

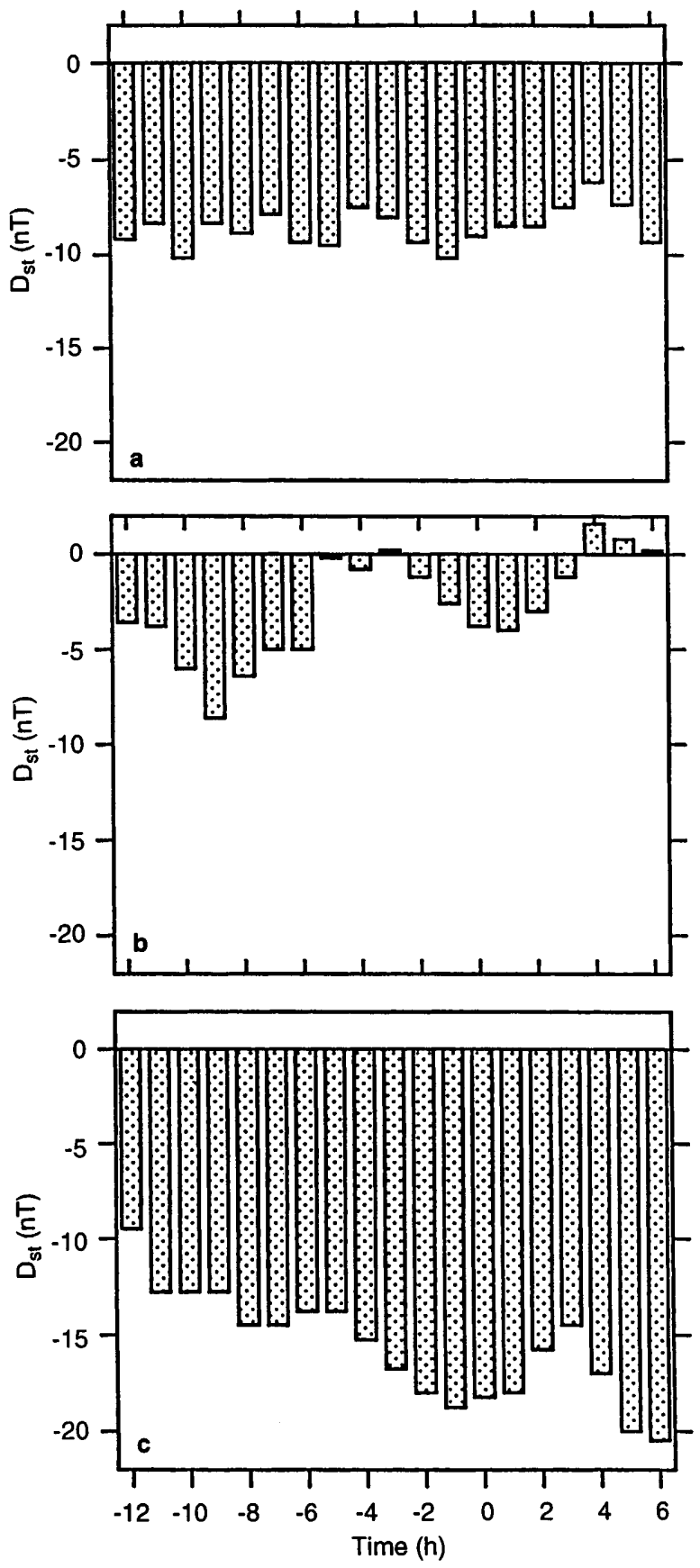

Fig. 5a-c. The results of a superposed epoch analysis of the Dst index during a the correlated (resonant) events described in Paper 1 (see main text), and the $\mathbf{b}$ dawn and $\mathbf{c}$ postnoon populations of uncorrelated waves discussed here

and reported by Yeoman et al. (1997) had a scale size of $\sim 60 \mathrm{~km}$ and the ground magnetic signature was strongly attenuated. This was a result of the phase mixing of very narrow (localised) resonant signature rather than it possessing a small azimuthal scale size (high $m$-number).

Estimations of the drift speed of protons in the equatorial plane have been made by Allan et al. (1982, 1983 ) using the observed period and $m$-value for a given wave. Yeoman et al. (1992) gave an expression for this:

$$
V_{e q}=\frac{2 \pi L R_{E}}{m \tau},
$$


where $V_{e q}$ is the drift velocity in the equatorial plane and $\tau$ is the wave period. $L$ is the $L$-shell of the drifting particles, $m$ is the azimuthal wave number and $R_{E}$ is the radius of the Earth. Particle populations with drift speeds in the range $20-40 \mathrm{~km} \mathrm{~s}^{-1}$ (consistent with the gradient curvature drift for protons with energies in the range $35-70 \mathrm{keV}$ ) were deduced to be the driving mechanism for the storm time Pc5s of Allan et al. (1982, 1983) and also to be consistent with the equatorward propagating Pc5s observed by Yeoman et al. (1992). Assuming a value of $V_{e q}$ of $30 \mathrm{~km} \mathrm{~s}^{-1}$, $L=6.6$ for Troms $\varnothing$ and $R_{E}=6400 \mathrm{~km}$ we obtain an expression relating the period of such waves to their expected $m$ values,

$$
m=\frac{8870}{\tau} .
$$

The 12 uncorrelated events presented here have wave frequencies from $4.2-17.9 \mathrm{mHz}$. If a particle population of similar energy to that for storm time Pc5s is assumed to be the source for the uncorrelated events, the corresponding $m$ values are, from Eq. (4), in the range, 37-159 (equivalent to azimuthal wavelengths at Tromsø $\left(69.6^{\circ} \mathrm{N}\right), \lambda_{\mathrm{az}}$, in the range $\left.86-372 \mathrm{~km}\right)$. These may be compared to those given by Allan et al. $(1982,1983)$ for the storm-time Pc5s observed by the STARE radar, which ranged from 7-80 $\left(\lambda_{\mathrm{az}}=171-1964 \mathrm{~km}\right)$, and for the SABRE observations of equatorward propagating Pc5s presented by Yeoman et al. (1992) where $m$ ranged from 5-36 $\left(\lambda_{\mathrm{az}}=382-2750 \mathrm{~km}\right)$, for the 26 events that were studied quantitatively. In addition, the $m$ numbers exhibited by $\mathrm{Pg}$ pulsations are typically in the range 20 $40\left(\lambda_{\mathrm{az}}=343-685 \mathrm{~km}\right.$; e.g. Chisham et al., 1997).

\section{Summary}

The DOPE HF Doppler sounder, designed and deployed to make measurements of the ionospheric signatures of ULF waves at high latitudes, has identified a type of wave which has no ground magnetic signature and is distinct from the low- $m$ (large scale size) field line resonances observations of which have already been presented in Paper 1. These uncorrelated waves are divided into two sub-classes, those which occur under quiet geomagnetic conditions and in the dawn sector and those observed during more active conditions in the postnoon sector. Many of the waves exhibit frequencies greater than the resonance fundamentals of local field lines.

It is suggested that these may be the signatures of high- $m$ ULF waves where the ground magnetic signature has been strongly attenuated as a result of the scale size of the waves. The dawn population demonstrates similarities to giant $(\mathrm{Pg})$ pulsations which tend to be resonant at higher harmonics on magnetic field lines and occur exclusively in the dawn sector. The waves occurring in the afternoon are believed to be related to the storm-time Pc5s previously reported in VHF radar data. Dst measurements support these observations by indicating that the dawn and dusk classes of waves occur respectively during geomagnetically quiet and more active intervals.

The DOPE sounder has a spatial resolution of the order of $4 \mathrm{~km}$ in the ionosphere which enables it to resolve small-scale waves which are generally not detectable by ground magnetometers. Estimating expected $m$-values for these waves, assuming they are particle driven and using the method of Allan et al. (1982, 1983) and Yeoman et al. (1992) indicates that these events may have azimuthal wave numbers in the range 37-159. The DOPE system utilises a routine ground-based method which supplements satellite techniques and is important since small-scale ULF waves are currently a subject of considerable theoretical study. In combination with other instrumentation in Scandinavia, this will allow the examination of the modes and ionospheric boundary conditions of smallscale waves.

Acknowledgements. The authors wish to thank the Royal Society for funding the DOPE project and the Particle Physics and Astronomy Research Council for support during the work presented here; Peter Chapman, Stuart Crooks and Michael Parsons, technical staff of the Radio and Space Plasma Physics group who were involved in the construction and deployment of the sounder; Michael Rietveld, Thorstein Blomstrand and Stein Eliassen at the EISCAT site in Ramfjordmoen for looking after the DOPE sounder and for returning data tapes to the UK; the Sodankylä Geophysical Observatory and Børre Holmeslet at the Tromsø Auroral Observatory for providing magnetometer data; and, in particular, Anastasia (Molly) Stockton-Chalk for her invaluable assistance in the preparation of this study.

Topical Editor K.-H. Glassmeier thanks W. Allan and A.D.M. Walker for their help in evaluating this paper.

\section{References}

Allan, W., and A. N. Wright, Large- $m$ waves generated by small- $m$ field line resonances via the nonlinear Kelvin-Helmholtz instability, J. Geophys. Res., 102, 19 927, 1997.

Allan, W., E. M. Poulter, and E. Nielsen, STARE observations of a Pc5 pulsation with large azimuthal wave number, J. Geophys. Res., 87, 6163, 1982.

Allan, W., E. M. Poulter, and E. Nielsen, Pc5 pulsations associated with ring current proton drifts: STARE radar observations, Planet. Space Sci., 31, 1279, 1983.

Annexstad, J. O., and C. R. Wilson, Characteristics of $\mathrm{Pg}$ micropulsations at conjugate points, J. Geophys. Res., 73, 1805, 1968.

Chapman, P. J., A cost effective frequency synthesizer for Doppler applications, Radio and Space Plasma Physics Group technical report number 60, Leicester University, UK, 1995.

Chapman, P. J., HF Doppler transmitter, Radio and Space Plasma Physics Group technical report number 64, Leicester University, UK, 1997a.

Chapman, P. J., HF Doppler receiver, Radio and Space Plasma Physics Group technical report number 63, Leicester University, UK, 1997b.

Chen, L., and A. Hasegawa, A Theory of long period magnetic pulsations, 1. steady state excitation of field line resonances, J. Geophys. Res., 79, 1024, 1974.

Chisham, G., and D. Orr, Statistical studies of giant pulsations (Pgs): harmonic mode, Planet. Space Sci., 39, 999, 1991.

Chisham, G., I. R. Mann, and D. Orr, A statistical study of giant pulsation latitudinal polarisation and amplitude variation, J. Geophys. Res., 102, 9619, 1997. 
Fenwick, R. C., and O. G. Villard, Continuous recordings of frequency variation of the WWV-20 signal after propagation over a $4000 \mathrm{~km}$ path, J. Geophys. Res., 67, 3249, 1960.

Fenrich, R. C., and J. C. Samson, Growth and decay of field line resonances, J. Geophys. Res., 102, 20 031, 1997.

Fenrich, R. C., J. C. Samson, G. Sofko, and R. A. Greenwald, ULF high- and low- $m$ resonances observed with the Super Dual Auroral Network, J. Geophys. Res., 100, 21 535, 1995.

Georges, T. M., Infrasound from convective storms: examining the evidence, Rev. Geophys. Space Phys., 11, 571, 1973.

Glassmeier, K.-H., Magnetometer array observations of a giant pulsation event, J. Geophys., 48, 127, 1980.

Green, C. A., Observations of Pg pulsations in the northern auroral zone and at lower latitude conjugate regions, Planet. Space Sci., 27, 63, 1979.

Greenwald, R. A., W. Weiss, E. Nielsen, and N. P. Thomson, STARE: a new radar auroral backscatter experiment in northern Scandinavia, Radio Sci., 13, 1021, 1978.

Hughes, W. J., Hydromagnetic waves in the magnetosphere, in Solar Terrestrial Physics Ed. Carovillano, R. L., and Forbes, J. M., Reidel, Dordrecht, 1983.

Hughes, W. J., and D. J. Southwood, The screening of micropulsation signals by the atmosphere and ionosphere, J. Geophys. Res., 81, 3234, 1976.

Hughes, W. J., R. L. McPherron, J. N. Barfield, and B. H. Mauk, A compressional Pc4 pulsation observed by three satellites in geostationary orbit near local midnight, Planet. Space Sci., 27, 821,1979

Jacobs, J. A., and T. Watanabe, Doppler frequency changes in radio waves propagating through a moving ionosphere, Radio Sci., 1, 257, 1966.

Jacobs, J. A., Y. Kato, S. Matsushita, and V. A. Troitskaya, Classification of geomagnetic micropulsations, J. Geophys. Res., 69, 180, 1964.

Jarvis, M. J., and H. Gough, Digital ionosonde observations of Pc3-4 pulsations across the plasmapause, Planet. Space Sci., 36, $733,1988$.

Klostermeyer, J., and J. Röttger, Simultaneous geomagnetic and ionospheric oscillations caused by hydromagnetic waves, Planet. Space. Sci., 24, 1065, 1976.

Kokubun, S., Statistical characteristics of Pc5 waves at geostationary orbit, J. Geomagn. Geoelectr., 37, 759, 1985.

Kremser, G., A. Korth, J. A. Fejer, B. Wilken, A. V. Gurevich, and E. Amata, Observations of quasi-periodic flux variations of energetic ions and electrons associated with Pc5 geomagnetic pulsations, J. Geophys. Res., 86, 3345, 1981.

Lühr, H., The IMAGE magnetometer network, STEP Int. Newsl., 4, 4, 1994

Mao Tian, T. K. Yeoman, M. Lester, and T. B. Jones, Statistics of Pc5 pulsation events observed by SABRE, Planet. Space Sci., 39, 1239, 1991.

Mann, I. R., An MHD model for driven high $m$ field line resonances, Geophys. Res. Lett., 25, 1515, 1998.

Menk, F. W., Characterization of ionospheric Doppler oscillations in the Pc3-4 and Pi2 pulsation frequency range, Planet. Space Sci., 40, 495, 1992.

Nielsen, E., W. Guttler, E. C. Thomas, C. P. Stewart, T. B. Jones, and A. Hedburg, SABRE: a new radar auroral backscatter experiment, Nature, 304, 712, 1983.

Orr, D., Magnetospheric hydrodynamic waves: their eigenperiods, amplitudes and phase variations: a tutorial introduction, J. Geophys., 55, 76, 1984.

Poole, A. W. V., P. R. Sutcliffe, and A. D. M. Walker, The relationship between ULF geomagnetic pulsations and iono- spheric Doppler oscillations: derivation of a model, J. Geophys. Res., 93, 14 656, 1988.

Poulter, E. M., W. Allan, J. G. Keys, and E. Nielsen, Plasma trough ion mass densities determined from ULF pulsation eigen periods, Planet. Space Sci., 32, 1069, 1984.

Reddi, C. R., and B. R. Rao, Anomalous variations in phase path records, J. Atmos. Terr. Phys., 29, 1063, 1967.

Rishbeth, H., and P. J. S. Williams, The EISCAT ionospheric radar: the system and its early results, $Q$. J. R. Astron. Soc., 26, 478, 1985.

Ruohoniemi, J. M., R. A. Greenwald, K. B. Baker, and J. C. Samson, HF radar observations of Pc5 field line resonances in the midnight/early morning MLT sector, J. Geophys. Res., 96, $15697,1991$.

Southwood, D. J., Some features of field line resonances in the magnetosphere, Planet. Space Sci., 22, 483, 1974.

Sutcliffe, P. R., Modelling the ionospheric signatures of geomagnetic pulsations, J. Geomagn. Geoelectr., 46, 1011, 1994.

Sutcliffe, P. R., and A. W. V. Poole, Low latitude Pc3 pulsations and associated ionospheric oscillations measured by a digital chirp ionosonde, Geophys. Res. Lett., 11, 1172, 1984.

Sutcliffe, P. R., and A. W. V. Poole, Ionospheric Doppler and electron velocities in the presence of ULF waves, J. Geophys. Res., 94, 13 505, 1989.

Sutcliffe, P. R., and A. W. V. Poole, The relationship between ULF geomagnetic pulsations and ionospheric Doppler oscillations: model predictions, Planet. Space Sci., 38, 1581, 1990.

Tedd, B. L., K. D. Cole, and P. L. Dyson, The association between ionospheric and geomagnetic pulsations in the Pc3-4 range at mid-latitudes, Planet. Space Sci., 37, 1079, 1989.

Toman, K., Ionospheric phase- and group-path, J. Atmos. Terr. Phys., 29, 1019, 1967.

Walker, A. D. M., R. A. Greenwald, W. F. Stuart, and C. A. Green, STARE auroral radar observations of Pc5 geomagnetic pulsations, J. Geophys. Res., 84, 3373, 1979.

Walker, A. D. M., J. M. Ruohoniemi, K. B. Baker, R. A. Greenwald, and J. C. Samson, Spatial and temporal behaviour of ULF pulsations observed by the Goose Bay HF radar, J. Geophys. Res., 97, 12 187, 1992.

Wright, A. N., Dispersion and wave coupling in inhomogeneous MHD waveguides, J. Geophys. Res., 99, 159, 1994.

Wright, D. M., HF Doppler observations of ULF waves: system development and high-latitude results, PhD Thesis, University of Leicester, UK, August 1996.

Wright, D. M., T. K. Yeoman, and P. J. Chapman, High-latitude HF Doppler observations of ULF waves: 1. waves with large spatial scale sizes, Ann. Geophysicae, 15, 1548, 1997.

Wright, D. M., T. K. Yeoman, and J. A. Davies, A comparison of EISCAT and HF Doppler observations of a ULF wave, Ann. Geophysicae, in press, 1998.

Yeoman, T. K., M. Lester, D. Orr, and H. Lühr, Ionospheric boundary conditions of hydromagnetic waves: the dependence on azimuthal wave number and a case study, Planet. Space Sci., 38, 1315, 1990.

Yeoman, T. K., Mao Tian, M. Lester, and T. B. Jones, A study of Pc5 hydromagnetic waves with equatorward phase propagation, Planet. Space Sci., 40, 797, 1992.

Yeoman, T. K., D. M. Wright, T. R. Robinson, J. A. Davies, and M. T. Rietveld, High spatial and temporal resolution observations of an impulse-driven field line resonance in radar backscatter artificially generated with the Tromsø heater, Ann. Geophysicae, 15, 634, 1997. 